\title{
Cyclotron localization in a sub-10-nm silicon quantum dot single electron transistor
}

\author{
M. C. Lin $^{\text {a) }}$ \\ Graduate Institute of Electronics Engineering and Department of Electrical Engineering, \\ National Taiwan University, Taipei 106, Taiwan \\ K. Aravind ${ }^{\mathrm{a})}$ \\ Department of Physics, National Taiwan University, Taipei 106, Taiwan \\ C. S. $\mathrm{Wu}^{\mathrm{a})}$ \\ Graduate Institute of Electro-Optical Engineering and Department of Electrical Engineering, \\ National Taiwan University, Taipei 106, Taiwan \\ Y. P. Wu \\ Institute of Physics, Academia Sinica, Taipei 115, Taiwan \\ C. H. Kuan \\ Graduate Institute of Electronics Engineering and Department of Electrical Engineering, \\ National Taiwan University, Taipei 106, Taiwan \\ Watson Kuo \\ Department of Physics, National Chung Hsing University, Taichung 402, Taiwan \\ C. D. Chen ${ }^{\text {b) }}$ \\ Institute of Physics, Academia Sinica, Taipei 115, Taiwan
}

(Received 28 September 2006; accepted 8 December 2006; published online 16 January 2007)

The authors have fabricated and measured a lateral $\mathrm{Si}$ single electron transistor consisting of a succession of a big island and small quantum dots. The big island gives rise to a small period Coulomb oscillation riding on the large irregular oscillation arising from the small quantum dots. The peaks of the latter shift in the presence of a magnetic field, which is analyzed in the context of field-induced Landau level shift with a soft-wall confinement potential. Furthermore, the current peak was suppressed for fields beyond a threshold value. An explanation based on cyclotron localization at noninteracting Landau levels is presented. (C) 2007 American Institute of Physics.

[DOI: $10.1063 / 1.2431565]$

Quantum dot single electron transistors (QD-SETs) provide an interesting playground for unraveling the rich physics that exists on a mesoscopic scale. ${ }^{1}$ Semiconductor quantum dots nicknamed "artificial atoms" provide a discrete energy level spectrum. When incorporated with charging effect in the form of QD-SETs, they are capable of probing electron dynamics through quantized levels and are all the more exciting and fascinating. Further, QD systems form a unique platform for studying the impact of magnetic fields on electron motion and on discrete electronic levels. ${ }^{3}$ For the case of dimensionally confined GaAs and its family of heterostructures, this has been previously studied. ${ }^{4,5}$ For the case of silicon based QD devices, studies pioneered by researchers at Princeton have revealed a meticulous shift in the Coulomb peak position. ${ }^{6}$ The contribution of this work to the existing standpoint is the observation of a clear suppression of several current peaks in addition to the previously wellunderstood peak shift ${ }^{7}$ arising in the presence of magnetic fields. In this letter, we present and model our electron transport data for an electron-beam lithographically patterned $\mathrm{Si}$ QD-SET measured at $42 \mathrm{mK}$ in the presence of a varying external field (up to $\pm 5 \mathrm{~T}$ ). The electronic level structure of a silicon QD is more complicated than that of GaAs because of

\footnotetext{
a) Also at: Institute of Physics, Academia Sinica, Taipei 115, Taiwan.

b) Author to whom correspondence should be addressed; also at: Department of Electrical Engineering, National Chung Hsing University, Taichung 402, Taiwan; electronic mail: chiidong@phys.sinica.edu.tw
}

its multivalley structure at the $\Gamma$ point, and theoretical understanding 8,9 of electronic levels is very important to the modeling of transport phenomena.

The device structure is shown in the inset of Fig. 1 and fabrication details are provided in the Electronic physics auxiliary publication service (EPAPS) depository.

The room temperature $I-V_{b}$ characteristic is linear, while at lower temperatures there is manifestation of Coulomb

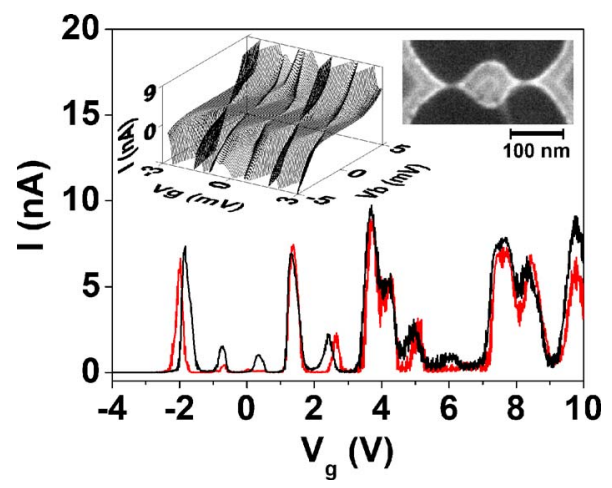

FIG. 1. (Color online) Oscillatory current modulation in gate voltage at $B=0 \mathrm{~T}$ (black curve) and $B=5 \mathrm{~T}$ (red curve) measured at $70 \mathrm{mK}$ for the device shown in the top-right inset. The device contains a big island connected to leads via small dots present in the nanoconstrictions. The top-left inset presents $I-V_{b}$ curves at ramping $V_{g}$ clearly marking the Coulomb blockade diamond; a closer look reveals fine wiggles arising from Coulomb oscillation in the big island. 

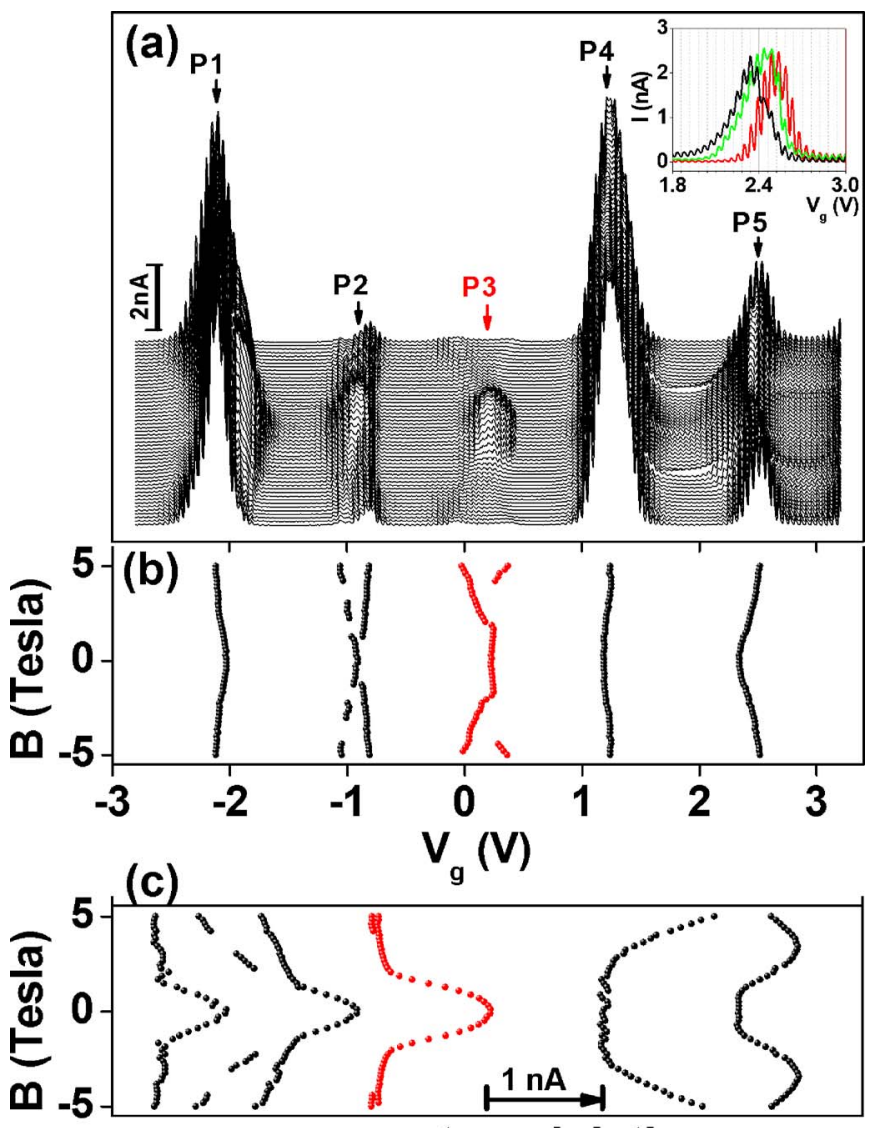

current modulation

FIG. 2. (Color online) (a) Dynamics of five current peaks in magnetic field at a bias voltage of $1.8 \mathrm{mV}$. The curves shift with field from $-5 \mathrm{~T}$ at the bottom to $+5 \mathrm{~T}$ at the top. The current peaks at zero magnetic field are clearly suppressed as seen from peak P3. The inset presents enlarged view of P5 describing the evolution of current peak position with the applied fields of $0 \mathrm{~T}$ (black curve), $2.5 \mathrm{~T}$ (green curve), and $5 \mathrm{~T}$ (red curve). Note also that the small wiggles show no shift in the field. The peak position shift in (b) and height modulation in (c) as a function of magnetic field for the five peaks are extracted from the raw data shown in (a) for further analysis. Peaks 2 and 3 show clear splitting in the presence of field, and the peaks shift in opposite directions. The peak height curves in (c) are placed in accordance with the respective positions in (a), and the magnitude of modulation is presented in the horizontal axis with a $1 \mathrm{nA}$ scale bar.

blockade. In addition, monotonic dependence of current on gate voltage was observed, a typical signature of a generic $n$-type field effect transistor originating from the proportional dependence of carrier concentration on gate voltage. At temperatures below $\sim 100 \mathrm{~K}$, a precursor to the periodic suppression of current emerged due to the charging effect, and this became more pronounced as the temperature was lowered. The main panel of Fig. 1 shows current modulations measured at $70 \mathrm{mK}$. At temperatures below about $20 \mathrm{~K}$, additional small oscillatory wiggles riding on the large current envelopes appeared, as can be seen in the inset of Fig. 2(a). The small wiggles are due to the big island, while the large envelope is due to the small dots embedded in the narrow constrictions. Here, the small dots are probing the charge states on the big island and the large magnitude of the wiggles along the steep edge indicates high charge sensitivity. The same figure also shows a shift in the peak position in $0,2.5$, and $5 \mathrm{~T}$ of external magnetic fields. Note that while there is no marked influence of an external magnetic field on the positions of wiggles contributed by the big island, the peak positions of the envelop contributed by the small dots Downloaded 04 Mar 2009 to 140.112.113.225. Redistribution subje
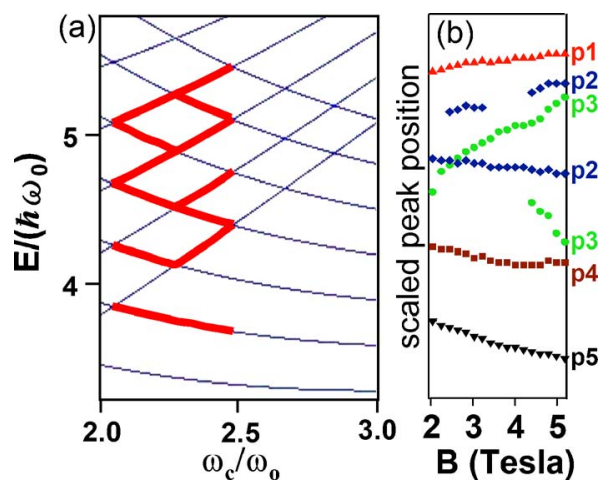

FIG. 3. (Color online) Comparison between (a) the theoretical eigenenergy spectrum in the presence of external field and (b) the experimental current peak position data. The zero field regions have multifold degeneracy and the beginning of our comparison with the theoretical curves from $B>2 \mathrm{~T}$ and $\omega_{c} / \omega_{0}>2$ is justified.

shifted with the magnetic fields. As there is no magnetic field effect on the Coulomb oscillations, the shifts in peak positions are attributed to factors other than simple charging effects in the small dots.

Figure 2 shows current modulation for a selected gate voltage span for five peaks and the peak shown in the inset is marked as P5 here. This gate voltage dependence can be simulated by a model circuit consisting of a big island in series with two small dots on each side. Together with the Coulomb diamond structure in current-voltage characteristics $\left(I-V_{b}\right)$ at ramping gate voltages (see the inset of Fig. 1), this simulation can provide an estimate of the sample parameters, including capacitances of each tunnel junction and the gate capacitances for the big island and small QDs. With this method, the charging energies for the large island and the two small dots were then estimated to be 24,83 , and $95 \mathrm{~K}$, respectively.

Figure 3 also shows the effect of a perpendicular magnetic field on the gate voltage modulation of these current peaks for fields between -5 and $+5 \mathrm{~T}$. In contrast with the two-dimensional electron gas systems, where oscillations are quite fragile, ${ }^{10}$ the features shown here were reproducible, fully symmetric on field reversal, and could withstand thermal cycling. A closer look at current modulations shows that peak positions actually shift with the magnetic fields. The amount of shift in chemical potential can be measured by scaling the gate voltage period with charging energy using the scaling factor $C_{g} / C \Sigma_{\Sigma} \approx 0.007$, where $C_{\Sigma}$ is the sum capacitance seen by the small dot and was found, in this case, to be of order $0.2 \mathrm{meV} / \mathrm{T}$, which is much greater than the shift given by Zeeman splitting $g^{*} \mu_{B} / 2$ of $0.06 \mathrm{meV} / \mathrm{T}$, where $g^{*}=2$ is the $g$ factor for $\mathrm{Si}$, and $\mu_{B}=e \hbar / 2 m_{e}$ is the Bohr magneton. The influence of magnetic fields on the energy levels of quantum dots can also be understood within the simple model described by or two-dimensional harmonic oscillator potential $V(t)=m \omega_{0}^{2} r^{2} / 2$. Here, the energy eigenvalue spectrum is given by ${ }^{3,11}$

$$
E_{n m}=\frac{1}{2}(n-m) \hbar \omega_{c}+\frac{1}{2}(n+m-1) \hbar\left(\omega_{c}^{2}+4 \omega_{0}^{2}\right)^{1 / 2},
$$

where $n, m=1,2, \ldots$ are the number of magnetic flux quanta $(h / e)$ enclosed by the electron orbit during cyclotron motion (called Landau index ${ }^{12}$ ) and angular moment, respectively. The cyclotron frequency is $\omega_{c}=e B / m^{*}, B$ is the applied field, to AIP license or copyright; see http://apl.aip.org/apl/copyright.jsp 
$e$ is the electron charge, and effective mass $m^{*}=0.19 m_{e}$ for $\mathrm{Si}(100)$. The energy spectrum of $E_{\mathrm{nm}} / \hbar \omega_{0}$ as a function of $\omega_{c} / \omega_{0}$ is shown in Fig. 3(a). Since the peak position in the gate voltage shown in Fig. 2(a) is the sum contribution from charging energy, random level spacing, and the energy eigenvalue spectrum $E_{\mathrm{nm}}$, and we are interested in only the $E_{\mathrm{nm}}$ term, the first two terms are thus removed in the figure by simply subtracting each peak position value by their corresponding zero-field values. Subtracting charging energy is justified by the constant interaction model of transport through quantum dots, where the peak position data can be translated to shifts in electrochemical potential. The charging energy scale $(95 \mathrm{~K})$ is used as a level arm to convert gate voltage to energy scale with a conversion factor $2 E_{C} / \Delta V_{g}$ $=15 \mathrm{meV} / \mathrm{V}$, and the deviation of this conversion, $8.25 \%$, is extracted from the successive intervals in Coulomb oscillation. The deviation represents the mean level spacing in the quantum dots. In addition, the peak position at zero field is equally spaced with $\hbar \omega_{0}=0.6 \mathrm{meV}$, as this is the proportionality constant between $E_{\mathrm{nm}}$ and the index $n$; the results for all five peaks are shown in Fig. 3(b). The $\hbar \omega_{0}$ value was estimated by comparing the measured shift with the spectrum shown in Fig. 3(a), and with the numerical calculation of Eq. (1). Figure 3(b) thus presents a qualitative comparison between the Landau level spectrum obtained from the spin independent eigenvalue equation and the trace of peak position with a sweeping magnetic field after accounting for the relevant charging energy scales. The cyclotron model described in Eq. (1) is only applicable when the cyclotron radius, $r_{c}=\hbar k_{F} / e B$ with $k_{F}$ the Fermi wave vector, is smaller than the radius of the dots. From Fig. 2(c) we estimate that this would correspond to a field of about $2 \mathrm{~T}$. The appropriate magnetic field window for the comparison of experimental and theoretical curves therefore begins from $2 \mathrm{~T}$ onwards. For this field, the $\hbar \omega_{c} / \hbar \omega_{0}$ is about 2 , and our device falls into the category of a soft-wall regime, which is defined as $\omega_{c} / \omega_{0}>1$. In this regime, the spacing between successive Landau levels can be expected to alter significantly with the magnetic field. This can probably explain the fact that the observed peak shifts do not exhibit the clear zigzag behavior seen in inverted GaAs/AlGaAs heterostructure experiments, ${ }^{13}$ where hard-wall confinement is more appropriate. In the case of Si-QDSETs (Ref. 6) with confinement strength of $\sim 100 \mathrm{meV}$, the peak position was found to shift linearly with the magnetic field as 0.3 and $0.6 \mathrm{meV} / \mathrm{T}$.

The current peaks P1-P5 have different heights and widths corresponding to different gate voltages. In Fig. 2(c), we show the trend in current modulation with magnetic field for all five peaks aligned with the peak positions. It can be seen that the first three peaks have the same behavior while the latter two have opposite characters. The former set undergoes a substantial suppression for fields greater than $2 \mathrm{~T}$. Above this field the electron wave function is strictly confined to the cyclotron radius, and the tunneling probability decreases exponentially with decreasing overlap between wave functions in the dots and leads. Despite the difference in peak height, they showed identical profiles described by a quadratic form with a coefficient of $0.21 \mathrm{nA} / \mathrm{T}^{2}$ within the turn-on field, reconfirming the cyclotron localization model. Above the turn-on field, P2 splits into two peaks of different heights. The lower peak was suppressed faster than the higher one because of the magnetic field induced localization being more effective. Peak 3 also splits into two, but with similar height and the same slope in height suppression with field. P4 and P5, however, showed no height change in the low field region. In higher fields, P4 increased monotonically while at P5 there was a further change of the slope, a similar behavior to that reported in Ref. 6 . The observed difference in height is attributed to the difference in the coupling strength $\Gamma$ of the discrete quantum levels to the chemical potentials of the leads. ${ }^{3}$ If we label the coupling strengths as $\Gamma_{1}-\Gamma_{5}$, we can see that $\Gamma_{1}>\Gamma_{4}>\Gamma_{5}>\Gamma_{2}>\Gamma_{3}$. Note that the pronounced cyclotron localization and complete current suppression thereof require very weak coupling to the source/ drain leads and isolation of the electron wave function, as in the case of P3. Conversely, the localization is incomplete in P1 and P4. The peak height changes reiterate our model of effective cyclotron localization in the small dots with soft-wall confinement. The contrary was demonstrated by Tilke et al.,${ }^{14}$ wherein no significant change in peak height was observed in the presence of an applied magnetic field, as the electrons were confined in a hard-wall potential $\left(\omega_{c} / \omega_{0}<1\right)$.

In summary, observation of electron cyclotron motion confined in a lateral silicon single electron transistor is presented. The applied magnetic field had no effect on the small wiggles and the heights and the peak positions of the envelopes were modulated. Shifts in the peak positions were explained by the theory of cyclotron motion induced Landau level shift, as described by Eq. (1) and Fig. 3(a). The suppression in peak height follows a quadratic form for fields lower than a threshold value corresponding to the onset of cyclotron localization. Analysis on the position shift and height suppression suggested that the quantum dots are in the soft-wall confinement regime. The energy scales such as charging energy, cyclotron localization energy, and confinement energy were estimated and found to be in agreement with the proposed model.

${ }^{1}$ D. Heiss, in Quantum Dots: Doorway to Nanoscale physics, Lecture Notes in Physics (Springer, Berlin, 2005), Vol. 667, pp. 97-130.

${ }^{2}$ M. Bayer, O. Stern, P. Hawrylak, S. Fafard, and A. Forchel, Nature (London) 405, 923 (2000).

${ }^{3}$ H. Grabert and M. H. Devoret, Single Charge Tunneling (Plenum, New York, 1992), Vol. 294, pp. 167-216.

${ }^{4}$ R. C. Ashoori, H. L. Stormer, J. S. Weiner, L. N. Pfeiffer, S. J. Pearton, K. W. Baldwin, and K. W. West, Phys. Rev. Lett. 68, 3088 (1992).

${ }^{5}$ B. Meurer, D. Heitmann, and K. Ploog, Phys. Rev. Lett. 68, 1371 (1992). ${ }^{6}$ L. P. Rokhinson, L. J. Guo, S. Y. Chou, and D. C. Tsui, Phys. Rev. Lett. 87, 166802 (2001); Phys. Rev. Lett. 88, 186801 (2002); Phys. Rev. B 63, 035321 (2001).

${ }^{7}$ L. P. Kouwenhoven, D. G. Austing, and S. Tarucha, Rep. Prog. Phys. 64, 701 (2001).

${ }^{8}$ Y. Hada and M. Eto, Phys. Rev. B 68, 155322 (2003).

${ }^{9}$ K. Natori, T. Uehara, and N. Sano, Jpn. J. Appl. Phys., Part 1 39, 2550 (2000).

${ }^{10}$ B. J. van Wees, L. P. Kouwenhoven, C. J. P. M. Harmans, J. G. Williamson, C. E. Timmering, M. E. I. Broekaart, C. T. Foxon, and J. J. Harris, Phys. Rev. Lett. 62, 2523 (1989).

${ }^{11}$ V. Fock, Z. Phys. 47, 446 (1928).

${ }^{12}$ L. L. Sohn, L. P. Kouwenhoven, and G. Schön, Mesoscopic Electron Transport (Kluwer, Dordrecht, 1997), Vol. 345, p. 177.

${ }^{13}$ P. L. McEuen, E. B. Foxman, U. Meirav, M. A. Kastner, Y. Meir, N. S. Wingreen, and S. J. Wind, Phys. Rev. Lett. 66, 1926 (1991).

${ }^{14}$ A. T. Tilke, F. C. Simmel, R. H. Blick, H. Lorenz, and J. P. Kotthaus, Prog. Quantum Electron. 25, 97 (2001); J. Appl. Phys. 90, 942 (2001). 